\title{
CaVi - Simulation and Model Checking for Wireless Sensor Networks
}

\author{
A. Boulis ${ }^{1}$, A. Fehnker ${ }^{1}$, M. Fruth ${ }^{2, *}$ and A. McIver ${ }^{3}$ \\ ${ }^{1}$ National ICT Australia ${ }^{\dagger}$, Australian Technology Park, Locked Bag 9013, Alexandria NSW 1435, Australia \\ ${ }^{2}$ Oxford University Computing Laboratory, Wolfson Building, Parks Road, Oxford OX1 3QD, UK \\ ${ }^{3}$ Macquarie University, Department of Computing, Macquarie University NSW 2109, Australia
}

\begin{abstract}
CaVi provides a uniform interface to state-of-the-art simulation methods and formal verification methods for wireless sensor network. Simulation is suitable to examine the behavior of a wireless sensor network in great detail. Due to the probabilistic behavior of these systems, however, the simulation covers only a small fraction of all possible behaviors. Formal model checking techniques, based on Markov Decision Processes, use less detailed and more abstract models and compute exact probabilities and expected values for the entire behavior, where simulation can only give averages. CaVi allows for creating a single model for simulation, Monte-Carlo simulation, and model checking.
\end{abstract}

\section{Introduction}

$\mathrm{CaVi}$ provides a unified modelling interface to the stateof-the-art simulator Castalia, and model checking tool PRISM. Castalia is an open-source, publicly-available, wireless sensor network simulator, developed at National ICT Australia [4]. PRISM is an established tool for probabilistic model checking, developed at the University of Oxford, UK [2]. Inspired by other graphical tools [3], we propose a graphical-style of specification that acts as a modelling environment for simulation and model checking.

\section{Wireless Network Simulator Castalia}

Castalia is a Wireless Sensor Network (WSN) simulator based on the OMNet++ platform that can be used by researchers and developers who want to test their distributed algorithms and protocols within a realistic wireless channel and radio model which takes account of the physical characteristics of the radio [4]. Castalia can also be used to

\footnotetext{
*This work was in part supported by the EPSRC grant EP/D076625/2

†Funded through the Australian Government's Backing Australia's Ability initiative, in part through the Australian Research Council.
}

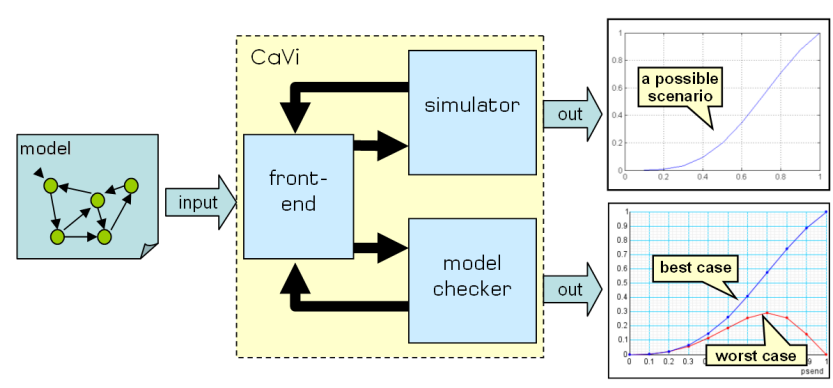

evaluate different platform characteristics for specific applications, since it is highly tunable, and can simulate a wide range of platforms. The main features of Castalia are:

- Advanced channel/radio model based on empirically measured data.

- Detailed state transition for the radio, allowing multiple transmission power levels.

- A highly flexible physical process model.

- Sensing device noise, bias, and power consumption.

- Node clock drift, CPU power consumption.

- Resource monitoring that goes beyond energy consumption (such as memory and CPU time).

- A highly configurable MAC layer protocol.

Castalia was designed right from the beginning so that the users can easily implement/import their algorithms and protocols into Castalia while making use of the features the simulator is providing. Proper modularization and a configurable, automated build procedure help towards this end.

\section{Formal Network Models in PRISM}

$\mathrm{CaVi}$ generates PRISM models automatically using generic templates based on flooding and gossiping protocols. The novelty is that the behavior of the wireless links is based on a empirically validated model proposed by Zuniga and Krishnamachari [5], the same model used in Castalia. This model takes into account the actual layout of the net- 


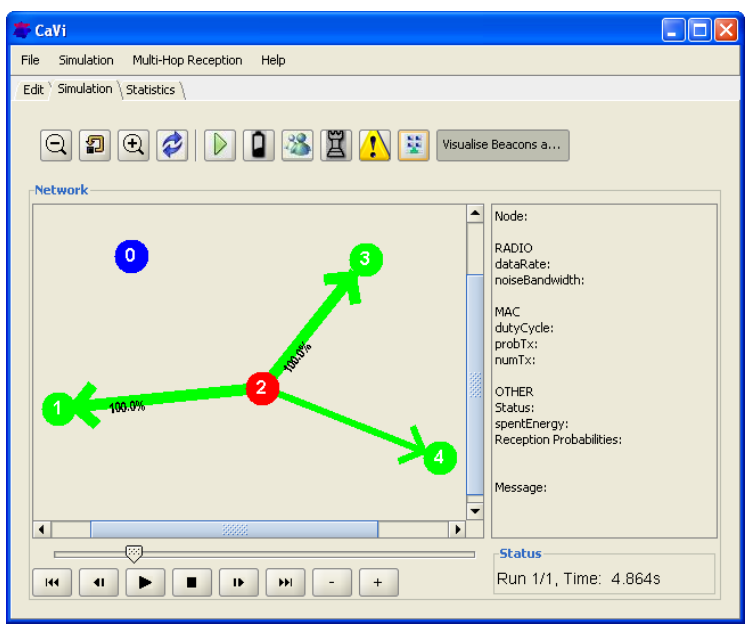

Figure 1. Visualizing an event trace.

work and the states of the surrounding network nodes, but also the modulation type and other network and radio parameters such as transmission power.

Once the PRISM input has been generated, PRISM transforms the model into a Discrete Time Markov Chain or a Markov Decision Process. These can then be analyzed exhaustively relative to a specified property using a suite of numerical algorithms. $\mathrm{CaVi}$ uses this analysis to compute multi-hop reception probabilities. The generated PRISM models can be either synchronous or asynchronous. The synchronous models assume that nodes receive or send at the same time, while the asynchronous models allows for an arbitrary delay between reception and transmission. The latter covers all possible interleavings; something that is difficult if not impossible to achieve by simulation. For a detailed description of the PRISM models see [1]. For models up to 20 nodes PRISM computes the exact multi-hop reception probabilities rather than approximate averages, and this typically faster than Monte Carlo simulation could do.

\section{$4 \mathrm{CaVi}$}

Creating and Editing a Network. CaVi models a network as a collection of nodes distributed in the deployment area. As an aid to determine the proper spatial distribution of the nodes, $\mathrm{CaVi}$ can visualize the worst and best-case one-hop reception strength. Protocol, parameters and characteristics of the wireless medium then determine the network behavior, and can be changed per node, or network wide. CaVi models are saved as XML files, and can be exported to Castalia and PRISM.

Simulation and Visualization of Traces. CaVi can automatically generate a Castalia model, simulate it, and visual-

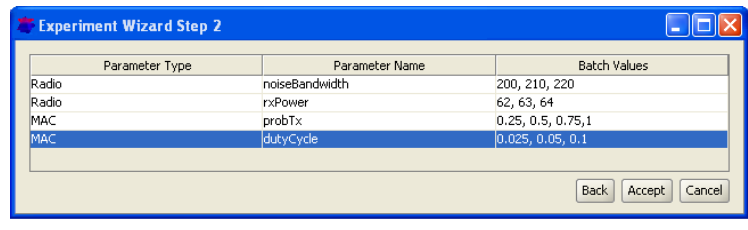

Figure 2. Set-up for Monte-Carlo simulation.

ize the trace. $\mathrm{CaVi}$ can also import Castalia traces generated outside of $\mathrm{CaVi}$. CaVi shows which node sends a packet to which node when, with which link probability, and whether the transmission succeeded. In addition, $\mathrm{CaVi}$ can display application dependent information, such as the energy level, application messages, as well as warning messages, and for a tree spanning application how the tree is being constructed from the root node.

Monte-Carlo Simulation and Model Checking. $\mathrm{CaVi}$ supports Monte-Carlo simulations for parameter sensitivity analysis. Given arrays of parameter values, $\mathrm{CaVi}$ runs for each possible combination of parameter values one batch of simulations, and return the percentage of receptions, transmissions, and collisions. $\mathrm{CaVi}$ allows also to compute the multi-hop reception probability, using PRISM, for each batch.

\section{Future Work}

$\mathrm{CaVi}$ presently allows for modelling wireless networks graphically, simulation with Castalia, as well as computing multi-hop reception probabilities with PRISM. It is able to visualize results from simulation, Monte-Carlo simulation or model checking, for ranges of parameter values. The next step is to include more application dependent visualizations, and the ability to customize the visualization for new applications. Furthermore, $\mathrm{CaVi}$ will offer graphical tools to evaluate statistical information obtained from parameter analysis and Monte-Carlo simulation.

\section{References}

[1] A. Fehnker, M. Fruth, and A. McIver. Graphical modelling for simulation and formal analysis of wireless network protocols. In MeMot 2007. LNCS, 2008. Submitted for review.

[2] A. Hinton, M. Kwiatkowska, G. Norman, and D. Parker. PRISM: A tool for automatic verification of probabilistic systems. In TACAS'06, 2006.

[3] Opnet. Network simulator. http://www. opnet. com.

[4] H. Pham, D. Pediaditakis, and A. Boulis. From simulation to real deployments in WSN and back. In $t 2 p W S N$ 2007, 2007.

[5] M. Zuniga and B. Krishnamachari. Analyzing the transitional region in low power wireless links. In SECON 2004. IEEE, 2004. 